\title{
Физико-химические характеристики и механизм сорбции алюмосиликатом ионов некоторых металлов из водных растворов
}

\author{
® 2020 Грибанов Е.Н. \\ Орловский государственный университет имени И.С. Тургенева, Орёл \\ Поступила в редакцию 17.04.2020 г.
}

DOI: $10.17308 /$ sorpchrom.2020.20/3139

Тематика работы связана с изучением возможности сорбционного концентрирования и выделения из водных растворов некоторых токсичных металлов для последующего решения ряда экологических проблем, связанных с очисткой природных и сточных вод от ионов данных тяжёлых металлов.

Целью настоящей работы являлось систематическое изучение сорбции $\mathrm{Pb}(\mathrm{II}), \mathrm{Zn}(\mathrm{II}), \mathrm{Hg}(\mathrm{II})$, $\mathrm{Cd}(\mathrm{II}), \mathrm{Ni}(\mathrm{II}), \mathrm{As}(\mathrm{III})$ природным алюмосиликатом из водных растворов, определение химизма протекающих процессов и его термодинамических характеристик.

В работе систематически изучена сорбционная способность природного алюмосиликата по отношению к $\mathrm{Pb}(\mathrm{II}), \mathrm{Zn}(\mathrm{II}), \mathrm{Hg}(\mathrm{II}), \mathrm{Cd}(\mathrm{II}), \mathrm{Ni}(\mathrm{II})$ и $\mathrm{As}(\mathrm{III})$. Определены оптимальные условия сорбции данных элементов из водных растворов: кислотность среды и время контакта фаз. Показано, что степень извлечения $\mathrm{Cd}(\mathrm{II}), \mathrm{Hg}(\mathrm{II})$ и $\mathrm{As}(\mathrm{III})$ из модельных водных растворов в оптимальных условиях кислотности среды $>95 \%$, для $\mathrm{Zn}(\mathrm{II})$ и $\mathrm{Ni}(\mathrm{II})$ данный параметр составляет $85-89 \%$, а для $\mathrm{Pb}(\mathrm{II}) \sim 64 \%$. Сорбционное равновесие в системе «сорбент-элемент» устанавливается за 20-25 минут (цинк, никель, мышьяк) и 60-70 минут (ртуть). В изучаемом интервале концентраций металлов построены изотермы сорбции, которые можно отнести к Ленгмюровскому типу. Сорбционная емкость минерала составляет от 12.0 мг/г для $\mathrm{Hg}(\mathrm{II})$ до 38.9 мг/г для As(III). Коэффициенты распределения в оптимальных условиях сорбции металлов достигают $\sim \mathrm{n} \cdot 10^{3}$. Изменение энергии Гиббса при сорбции лежит в интервале от 19.8 до -24.8 кДж/моль. По данным ИК-спектроскопии сделано предположение о химизме протекающего процесса, основанного на ионном обмене.

Полученные результаты представляют интерес для лабораторий и организаций, занимающихся природоохранными технологиями, а также при сорбционном концентрировании и выделении металлов из объектов сложного химического состава.

Ключевые слова: сорбция, алюмосиликаты, токсичные металлы.

\section{Введение}

Ионы металлов поступая в объекты окружающей среды в результате антропогенной деятельности, способны накапливаться в отдельных звеньях трофической цепи и далее попадать в организмы животных и человека, оказывая на них пагубное воздействие вплоть до летального исхода [1-3]. Остро выглядит проблема загрязнения вод различной природы соединениями ртути, свинца, мышьяка, кадмия, цинка и никеля, что связано с их высокой токсичностью (первый и второй класс опасности [3]). Поступая в организм человека, они могут приводить к гипертонической болезни, почечной недостаточности, параличу, а также патологическим изменения в нервной, иммунной, пищеварительной системах и другое [2-4]. Необходима разработка эффективных способов извлечения данных металлов из вод. Хорошо зарекомендовал себя метод, основанный на сорбционном выделении токсикантов различной природы [5-8]. 
Основным требованиям к сорбционным материалам отвечают природные алюмосиликаты [9-12]. Они устойчивы к внешнему воздействию, обладаю высокими сорбционными свойствами по отношению к различным по природе катионам и при этом отличаются экономической доступностью.

Целью настоящей работы являлось систематическое изучение сорбции $\mathrm{Pb}(\mathrm{II})$, $\mathrm{Zn}(\mathrm{II}), \mathrm{Hg}(\mathrm{II}), \mathrm{Cd}(\mathrm{II}), \mathrm{Ni}(\mathrm{II}), \mathrm{As}(\mathrm{III})$ природным алюмосиликатом из водных растворов, определение химизма протекающих процессов и его термодинамических характеристик.

\section{Экспериментальная часть}

Растворы ионов металлов (10.0 мг/дм³ $)$ готовили из ГСО ионов свинца(II) (ГСО 7012), кадмия(II) (ГСО 7874), ртути(II) (ГСО 7343), мышьяка(III) (ГСО 7264), никеля (II) (ГСО 7785), цинка(II) (ГСО 7256) разбавлением дистиллированной водой.

В качестве сорбента использовали алюмосиликат Хотынецкого месторождения (химический состав приведен в [13]). Его предварительно истирали в фарфоровой ступке [14] и обдували потоком воздуха $\left(70 \pm 1^{\circ} \mathrm{C}\right)$ до воздушно-сухого состояния.

Контроль концентрации инов $\mathrm{Pb}(\mathrm{II})$ и $\mathrm{Cd}(\mathrm{II})$ проводили методом атомно-абсорбционной спектрометрии (спектрометр Квант-2А) в режиме прямой абсорбции с атомизацией в пламени «воздух/пропан - бутановая смесь», $\mathrm{Hg}$ (II) и $\mathrm{As}$ (III) определяли с использованием генератора ртутно-гидридного ГРГ-107 методом «холодного пара» с атомизацией в кварцевой кювете. Концентрации $\mathrm{Zn}(\mathrm{II})$ и $\mathrm{Ni}(\mathrm{II})$ контролировали спектрофотометрически (спектрофотометр СФ-56) по реакции с 4-(2-пиридилазо)резорцином.

ИК-спектры регистрировали в области 4000-450 $\mathrm{cm}^{-1}$ (ИК-фурье спектрометр ФСМ 2202) таблетированием с КВг.

Сорбцию изучали в статическом режиме при периодическом перемешивании при $20.0 \pm 1.0^{\circ} \mathrm{C}$. В раствор аналита с соответствующей концентрацией и объемом равным 25 мл вносили 0.4 г сорбента. Далее рассчитывали степень извлечения (R, \%) вещества по уравнению:

$$
R, \%=\frac{c_{0}-c}{c_{0}} \times 100,
$$

где $c_{0}$ - концентрация аналита в растворе до сорбции, мкг $/ \mathrm{cm}^{3} ; \mathrm{c}-$ концентрация аналита в растворе после сорбции, мкг/ $\mathrm{cm}^{3}$.

Концентрацию ионов металлов в водной фазе определяли по предварительно построенному градуировочному графику. Содержание аналита в фазе сорбента определяли по разности между их исходной и равновесной концентрацией в растворе. Из зависимости «R,\% - $\mathrm{pH}$ и «R, \% - время» находили оптимальный интервал кислотности среды и время при которых степень извлечения металлов достигает максимального значения.

\section{Обсуждение результатов}

Эффективность сорбционного извлечения ионов металлов из водных растворов определяется кислотностью среды, при которой степень извлечения достигает максимального значения, временем установления сорбционного равновесия и сорбционной емкостью к конкретному веществу.

Установлено, что степень извлечения изучаемых ионов металлов алюмосиликатом из модельных водных растворов достигает максимального значения в области 
$\mathrm{pH}$ близкой к нейтральной среде (рис. 1). Так для $\mathrm{Cd}(\mathrm{II}), \mathrm{Hg}(\mathrm{II})$ и $\mathrm{As}(\mathrm{III})$ она составляет $>95 \%$, для Zn(II) и $\mathrm{Ni}$ (II) 85-89\%, а для $\mathrm{Pb}$ (II) 64\%. Можно предположить, что степень извлечения ионов свинца снижается из-за проявления минералом «ситового эффекта», связанного с селективной сорбцией ионов, диаметр которых меньше или равен диаметру пор материала [15]. Так для $\mathrm{Pb}(\mathrm{II})$ соответствует бо́льший ионный радиус в сравнении с ионами остальных металлов, изучаемых в работе [16]. Установлено, что сорбционное равновесие в системе «сорбент-элемент» устанавливается за 20-25 минут для цинка, никеля и мышьяка, а для ртути за 60-70 минут.
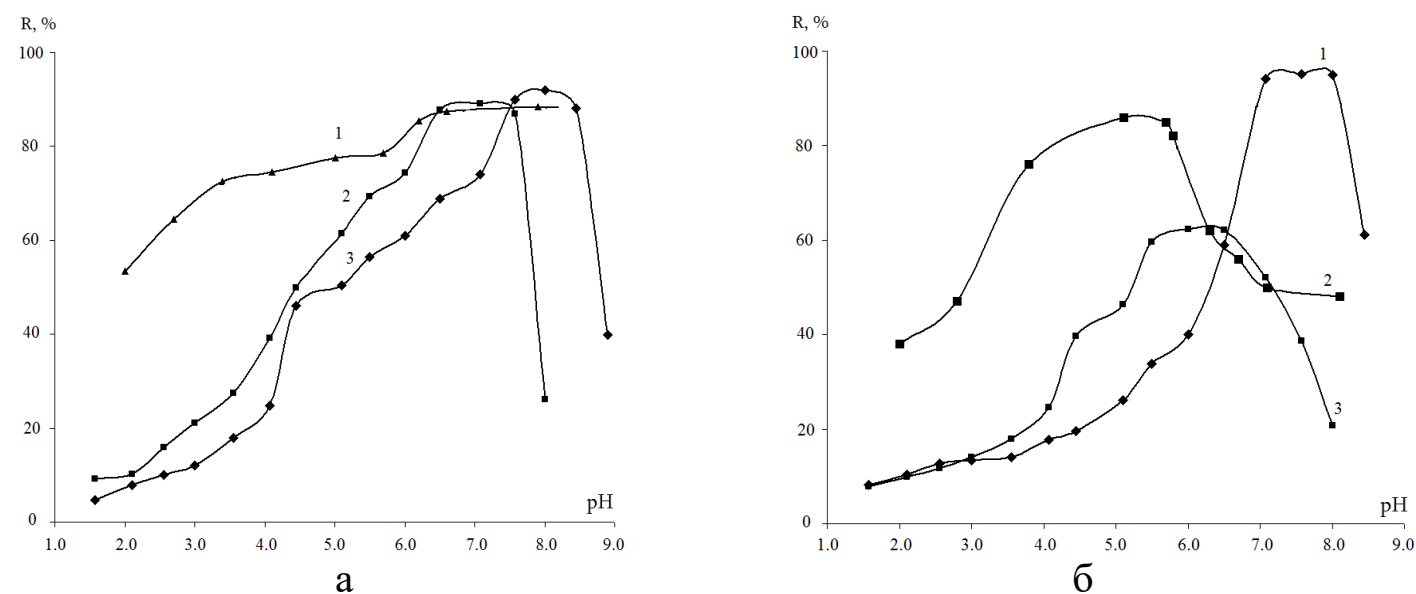

Рис. 1. Влияние кислотности среды на степень извлечения алюмосиликатом:

a) $1-\mathrm{Ni}(\mathrm{II}), 2-\mathrm{Cd}$ (II) и $3-\mathrm{As}(\mathrm{III})$; б) $1-\mathrm{Hg}(\mathrm{II}), 2-\mathrm{Zn}$ (II), $3-\mathrm{Pb}$ (II)

Fig. 1. Influence of medium acidity on the degree of extraction by aluminosilicate:

a) $1-\mathrm{Ni}(\mathrm{II}), 2-\mathrm{Cd}(\mathrm{II})$ and $3-\mathrm{As}(\mathrm{III})$; b) $1-\mathrm{Hg}(\mathrm{II}), 2 \mathrm{Zn}(\mathrm{II}), 3-\mathrm{Pb}$ (II)

Для всех изученных систем «алюмосиликат-металл» построены изотермы сорбции (рис. 2), которые имеют выпуклую форму с выходом на насыщение и удовлетворительно описываются уравнением изотермы Ленгмюра:

$$
a=a_{m} \cdot \frac{K \cdot c}{1+K \cdot c},
$$

где $a_{\mathrm{m}}-$ максимальная ёмкость монослоя, мг/г; $a$ - концентрация ионов металла в фазе сорбента, мг/г; $c$ - равновесная концентрация ионов металла в растворе после сорбции, мкг $/ \mathrm{cm}^{3} ; K-$ константа сорбционного равновесия.

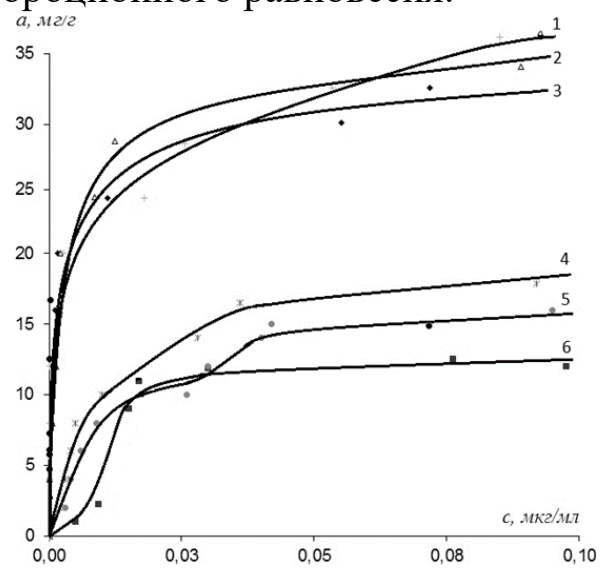

Рис. 2. Изотермы сорбции алюмосиликатом: 1 - $\mathrm{As}(\mathrm{III}), 2$ - $\mathrm{Pb}(\mathrm{II})$, $3-\mathrm{Cd}(\mathrm{II}), 4-\mathrm{Zn}(\mathrm{II}), 5-\mathrm{Ni}(\mathrm{II}), 6-\mathrm{Hg}(\mathrm{II})$

Fig. 2. Sorption isotherms for aluminosilicate: $1-\mathrm{As}(\mathrm{III}), 2-\mathrm{Pb}(\mathrm{II}), 3-\mathrm{Cd}(\mathrm{II})$,

$$
4-\mathrm{Zn}(\mathrm{II}), 5-\mathrm{Ni}(\mathrm{II}), 6-\mathrm{Hg}(\mathrm{II})
$$


По уравнению линейной зависимости, построенной в координатах $1 / a-1 / c$, определяли следующие физико-химические параметры: сорбционные емкости сорбентов $\left(a_{m}, \mathrm{Mг} / \Gamma\right)$ и константы адсорбционного равновесия. Изменение энергии Гиббса $(\Delta \mathrm{G})$ при сорбции определяли по уравнению: $\Delta \mathrm{G}=-R T \ln K(3)$.

Характеристики сорбции алюмосиликатом изучаемых в работе металлов систематизированы и представлены в таблице 1. Сорбционная емкость минерала в оптимальных условиях кислотности среды колеблется от 12.0 до 38.9 мг/г. Коэффициенты распределения в системе «сорбент-металл» достигают $\sim \mathrm{n} \cdot 10^{3}$.

Таблица 1. Характеристики сорбции металлов алюмосиликатом $\left(\mathrm{t}=20 \pm 1^{\circ} \mathrm{C}, \mathrm{n}=5\right)$ Table 1. Characteristics of metal sorption by aluminosilicate $\left(\mathrm{t}=20 \pm 1^{\circ} \mathrm{C}, \mathrm{n}=5\right)$

\begin{tabular}{|c|c|c|c|c|c|c|c|}
\hline $\begin{array}{c}\text { № } \\
\text { п/п }\end{array}$ & Металл & $\mathrm{pH}_{\text {опт }}$ & $\tau$, мин & $\begin{array}{c}a_{\mathrm{m},} \\
\mathrm{M} / \Gamma\end{array}$ & $\begin{array}{c}\mathrm{D} \cdot 10^{-3}, \\
\mathrm{~cm}^{3} / \Gamma\end{array}$ & $\mathrm{R}, \%$ & $\begin{array}{c}-\Delta \mathrm{G}_{293,} \\
\text { кДж/моль }\end{array}$ \\
\hline 1 & Ртуть & $7.0-8.0$ & $60-70$ & 12.0 & 0.65 & $95 \pm 3$ & 22.4 \\
\hline 2 & Свинец & $5.5-6.5$ & $40-50$ & 35,2 & 4.43 & $64 \pm 2$ & 24.8 \\
\hline 3 & Цинк & $5.0-6.0$ & $20-25$ & 16.4 & 1.43 & $85 \pm 2$ & 24.4 \\
\hline 4 & Никель & $6.5-7.8$ & $20-25$ & 15.2 & 1.30 & $89 \pm 2$ & 19.8 \\
\hline 5 & Мышьяк & $6.3-7.5$ & $20-25$ & 38,9 & 6.05 & $98 \pm 2$ & 23.9 \\
\hline 6 & Кадмий & $7.5-8.5$ & $35-40$ & 34,8 & 7.15 & $98 \pm 2$ & 20.6 \\
\hline
\end{tabular}

Изучена десорбция ионов изучаемых металлов с поверхности алюмосиликата. Десорбцию проводили водными растворами хлорида аммония, хлорида натрия, цитрат аммония, оксалата натрия и мочевины с концентрацией 5 моль/дм³. Выбор природы десорбирующих агентов связан с их способностью к ионному обмену с функциональными группами алюмосиликата или к образованию достаточно прочных комплексных соединений с ионами металлов. Установлено, что минимальная степень десорбции элементов 19-26\% наблюдается при обработке минерала хлоридом натрия. Наилучший результат 92-97\% получен при десорбции раствором мочевины при нагревании до $40 \pm 1^{\circ} \mathrm{C}$, что говорит о достаточно прочной связи ионов металлов с поверхностными функциональными группами алюмосиликата.

О предполагаемом химизме сорбции судили по данным ИК-спектроскопии, сравнивая полосы поглощения (п.п.) в ИК-спектрах алюмосиликата до и после сорбции каждого из ионов металлов $[15,17]$. ИК-спектр изучаемого в работе минерала описан нами ранее [13]. В нём присутствуют: полосы поглощения в области волновых чисел 450-625 и 900-1300 см-1, которые можно соотнести с колебаниями связей в тетраэдрах [(Al,Si)O 4 ]-каркаса; полосы с максимумами при 1089 и 1046 см$^{-1}$ и слабовыраженным плечом при $1202 \mathrm{~cm}^{-1}$, которые связаны с валентными и деформационными колебаниями Si-O-связей; п.п. при 472, 522 и 667 см$^{-1}$, связанные с колебаниями различного рода $\mathrm{Si}-\mathrm{O}-\mathrm{Al}, \mathrm{O}-\mathrm{Si}-\mathrm{O}, \mathrm{Si}-\mathrm{O}-\mathrm{H}$ групп. В спектре наблюдаются валентные колебания гидроксильных групп при 3627 и $3435 \mathrm{~cm}^{-1}$. Колебания гидроксо-групп, принадлежащих $\mathrm{Si}-\mathrm{OH}$ или $\mathrm{Si}-\mathrm{O}(\mathrm{H})-\mathrm{Al}$ проявляются при $880 \mathrm{~cm}^{-1}$, в то время как п.п. при $792 \mathrm{~cm}^{-1}$ относится к деформационным колебаниям Si-O-Si-связей кремнекислородного тетраэдра $\left[\mathrm{SiO}_{4}\right]^{4-}$.

Функциональные группы Si-OH, Al-OH, Si-O-Al алюмосиликата, а также атомы кислорода, находящиеся на его поверхности, могут участвовать в образовании соединений в результате взаимодействий с катионами металлов. ИК-спектры систем «сорбент-металл» приведены на рис. 3. Из них следует, что после сорбции ионов металла полоса поглощения минерала при $880 \mathrm{~cm}^{-1}$ смещается в область более высоких частот до $953 \mathrm{~cm}^{-1}$, что можно связать с изменением полярности связи Si-O. Появляются новые полосы поглощения при 1160 и $1128 \mathrm{~cm}^{-1}$. Данные изменения можно объ- 
яснить колебаниями связей «металл-ОН». Следовательно, в основе химизма протекающего процесса лежит ионный обмен. Его схема с учетом констант гидролиза изучаемых в работе металлов, выглядит следующим образом:

$$
\mathrm{m}\left\{(\mathrm{Al}, \mathrm{Si}-\mathrm{O})^{-} \mathrm{X}^{+}\right\}+\mathrm{M}(\mathrm{OH})^{\mathrm{n}+}=\mathrm{m}\left\{(\mathrm{Al}, \mathrm{Si}-\mathrm{O})^{-} \mathrm{Me}(\mathrm{OH})^{\mathrm{n}+}\right\}+\mathrm{mX}^{+},
$$

где $\mathrm{X}$ - обменный катион $\left(\mathrm{H}^{+}, \mathrm{K}^{+}\right), \mathrm{M}$ - металл.

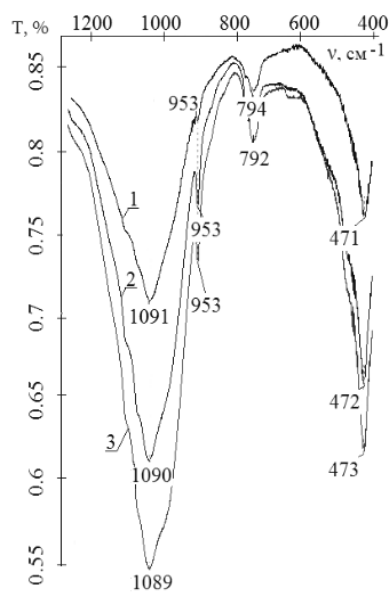

a

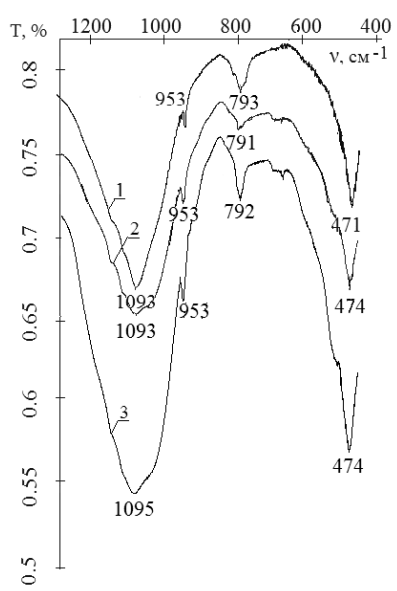

6

Рис. 3. ИК-спектр алюмосиликата после сорбции: а) 1 - Hg(II), $2-\mathrm{Pb}(\mathrm{II}), 3-\mathrm{Cd}(\mathrm{II})$; б) 1 - $\mathrm{As}(\mathrm{III}), 2$ - Zn(II), 3 - Ni(II)

Fig. 3. IR spectrum of aluminosilicate after sorption: a) $1-\mathrm{Hg}(\mathrm{II}), 2-\mathrm{Pb}(\mathrm{II})$, 3 - Cd(II); b) 1 - As(III), 2 - Zn(II), 3 - Ni(II)

\section{Заключение}

Таким образом, в работе систематически изучены сорбционные свойства природного алюмосиликата по отношению к $\mathrm{Pb}(\mathrm{II}), \mathrm{Ni}(\mathrm{II}), \mathrm{Hg}(\mathrm{II}), \mathrm{Cd}(\mathrm{II}), \mathrm{Zn}(\mathrm{II})$ и $\mathrm{As}(\mathrm{III})$. Определены оптимальные условия сорбции металлов: кислотность среды и время контакта фаз. Изменение энергии Гиббса данного процесса в зависимости от природы металла лежит в интервале от -19.8 до -24.8 кДж/моль. Построены изотермы сорбции, которые можно отнести к Ленгмюровскому типу - имеют выпуклую форму с выходом на насыщение. Определена сорбционная емкость минерала по каждому из токсикантов. Установлено, что коэффициенты распределения в оптимальных условиях сорбции металлов достигают $\sim \mathrm{n} \cdot 10^{3}$. По данным ИК-спектроскопии сделано предположение о вероятном химизме сорбции, основанного на ионном обмене. Показана достаточно высокая эффективность применения алюмосиликата при очистке вод различной природы, загрязненных водорастворимыми соединениями $\mathrm{Pb}(\mathrm{II}), \mathrm{Zn}(\mathrm{II}), \mathrm{Hg}$ (II), $\mathrm{Cd}(\mathrm{II}), \mathrm{Ni}(\mathrm{II})$ и $\mathrm{As}(\mathrm{III})$. Полученные результаты представляют интерес для лабораторий и организаций, занимающихся природоохранными технологиями, а также при сорбционном концентрировании и выделении металлов из объектов сложного химического состава

\section{Список литературы}

1. Bayader F.A., Wessal M.Kh., Ahmed M.A. et al. // Research J. Pharm. and Tech. 2018. Vol. 11 No 9. pp. 4035-4041.
2. Онищенко Г.Г., Новиков С.М., Рахманин Ю.А. и др. Основы оценки риска для здоровья населения при воздействии химических 
веществ, загрязняющих окружающую среду. М. НИИ ЭЧ и ГОС. 2002. 408 c.

3. Авцын А. П,, Жаворонков А. А., Риш М. А. и др. Микроэлементозы человека. М. Медицина. 1991. 496 с.

4. Петросян В.С., Шувалова Е.А. Химия и токсикология окружающей среды. М. БукиВеди. 2017. 640 с.

5. Везенцев А.И., Перистая Л.Ф., Нгуен Фук Као и др. // Сорбиионные и хроматографические прочессы. 2018. Т. 18. № 1. С. 43-51.

6. Ксеник Т.В., Юдаков А.А., Перфильев А.В. // Экология и промышиленность России. 2009. № 4. C. 19-21.

7. Наинг Линн Сoe, Зин Мое, Мин Тху и др. // Сорбиионные и хроматографические nроиессы. 2019. Т. 19. № 5. С. 574-580.

8. Милютин В.В., Некрасова Н.А., Рудских В.В. и др. // Сорбичинные и хроматографические проиессы. 2018. № 3. С. 365-372.

9. Wang S., Peng Y.// Chemical Engineering Journal. 2010. Vol .156. No 1. pp. 11-24.

10. Minmin L, Lian H, Beidou X. et al. // Applied Surface Science. 2013. Vol. 273. pp. 706716.
11. Sabry M.Sh., Aly S.D., Farahat S.M. // International Journal of Environmental Science and Development. 2012. Vol. 3. No 4. pp. 362367

12. Грибанов Е.Н., Оскотская Э.Р., Саунина И.В. // Сорбционные и хроматографические прочессы. 2018. № 3. С. 916-922.

13. Грибанов Е.Н., Оскотская Э.Р., Кузьменко А.П. // Конденсированные среды и межсазные гранищы. 2018. Т. 20. № 1. С. 4249.

14. Грибанов Е.Н., Оскотская Э.Р., Свенский С.И. //Ученые записки Орловского государственного университета. Серия: Естественные, технические и медииинские науки. 2013. T. 53. № 3. C. 121-123.

15. Рабо Дж. Химия цеолитов и катализ на цеолитах. Т.1. М. Мир. 1980. 506 с.

16. Лурье Ю.Ю. Справочник по аналитической химии. М. Химия. 1989. 448 c.

17. Mozgawa W., Handke M., Jastrzebski W. // Journal of Molecular Structure. 2004. Vol. 704. No 1-3. pp. 247-257.

\title{
Physicochemical characteristics and mechanism of sorption of some metal ions from aqueous solutions by aluminosilicate
}

\author{
(B) 2020 Gribanov E.N. \\ Turgenev Orel State University, Orel, Russia
}

\begin{abstract}
The subject of the study was the investigation of the possibility of sorption concentration and the isolation of some toxic metals from aqueous solutions for the subsequent solution of a number of environmental problems associated with the purification of natural and waste waters from the ions of these heavy metals.

The aim of this study was systematic study of the sorption of $\mathrm{Pb}$ (II), $\mathrm{Zn}(\mathrm{II}), \mathrm{Hg}(\mathrm{II}), \mathrm{Cd}(\mathrm{II}), \mathrm{Ni}(\mathrm{II})$, and As(III) by natural aluminosilicate from aqueous solutions, to determine the chemistry of the processes and its thermodynamic characteristics.

In the study the sorption capacity of natural aluminosilicate in relation to $\mathrm{Pb}(\mathrm{II}), \mathrm{Zn}(\mathrm{II}), \mathrm{Hg}(\mathrm{II}), \mathrm{Cd}(\mathrm{II})$, $\mathrm{Ni}(\mathrm{II})$, and As(III) was systematically investigated. The optimal conditions for the sorption of these elements from aqueous solutions were determined: the acidity of the medium and the phase contact time. It is shown that the degree of extraction of $\mathrm{Cd}(\mathrm{II}), \mathrm{Hg}(\mathrm{II})$, and $\mathrm{As}(\mathrm{III})$ from model aqueous solutions under optimal acidity of the medium was $>95 \%$, for $\mathrm{Zn}$ (II) and $\mathrm{Ni}$ (II) this parameter was $85-89 \%$, and for $\mathrm{Pb}$ (II) it was $\sim 64 \%$. Sorption equilibrium in the "sorbent-element" system was established in 20-25 min (zinc, nickel, arsenic) and 6070 min (mercury). In the studied range of metal concentrations, sorption isotherms were plotted, which can be attributed to the Langmuir type. The sorption capacity of the mineral ranged from $12.0 \mathrm{mg} / \mathrm{g}$ for $\mathrm{Hg}$ (II) to 38.9 $\mathrm{mg} / \mathrm{g}$ for As(III). The distribution coefficients under optimal conditions of metal sorption reach $\sim \mathrm{n} \cdot 10^{3}$. The change in the Gibbs energy during sorption was in the range from $-19.8 \mathrm{~kJ} / \mathrm{mol}$ to $-24.8 \mathrm{~kJ} / \mathrm{mol}$. Based on the IR spectroscopy data, an assumption about the chemistry of the process based on ion exchange was made.
\end{abstract}


The obtained results are of interest for laboratories and organisations dealing with environmental technologies, as well as for the sorption concentration and separation of metals from objects with complex chemical compositions.

Keywords: sorption, aluminosilicates, toxic metals.

\section{References}

1. Bayader F.A., Wessal M.Kh., Ahmed M.A. et al., Research J. Pharm. and Tech., 2018, Vol. 11, No 9, pp. 4035-4041.

2. Onishchenko G.G, Novikov S.M., Rakhmanin $\mathrm{Yu}$.A. et al. Basics of assessing the risk to public health when exposed to chemicals that pollute the environment, M., Nil ECH and GOS, 2002, 408 p.

3. Avtsyn A.P., Zhavoronkov A.A., Rish M. A. et al., Human microelementoses, M., Medicine, 1991, $496 \mathrm{p}$.

4. Petrosyan V.S., Shuvalova E.A., Chemistry and toxicology of the environment, M., BukiVedi, 2017, 640 p.

5. Vezentsev Al, Peristaya LF, Nguyen Fook Kao et al., Sorbtsionnye i khromatograficheskie protsessy. 2018, No 18, pp. 43-51.

6. Ksenik T.V., Yudakov A.A., Perfilyev A.V., Ecology and Industry of Russia, 2009, No 4, pp. 19-21.

7. Naing Lin Soe, Zin Moe, Min Thu et al., Sorbtsionnye i khromatograficheskie protsessy. 2019, Vol. 19, No 5, pp. 574-580.

8. Milyutin V.V., Nekrasova N.A., Rudskikh V.V. et al., Sorbtsionnye i khromatograficheskie protsessy, 2018, Vol. 18, No 3, pp. 365-372.

Грибанов Евгений Николаевич - к.Х.Н., доцент кафедры химии, Орловский государственный университет имени И.С. Тургенева, Орел
9. Wang S., Peng Y., Chemical Engineering Journal, 2010, Vol. 156, No 1, pp. 11-24.

10. Minmin L., Lian H, Beidou X., Ying Z., Applied Surface Science, 2013, Vol. 273, pp. 706-716.

11. Sabry M.Sh., Aly S.D., Farahat S.M., International Journal of Environmental Science and Development, 2012, Vol. 3, No 4, pp. 362367.

12. Gribanov E.N., Oskotskaya E.R., Saunina I.V., Sorbtsionnye $i$ khromatograficheskie protsessy. 2018, No 3, pp. 916-922.

13. Gribanov E.N., Oskotskaya E.R., Kuzmenko A.P., Condensed media and interfaces, 2018, Vol. 20, No 1, pp. 42-49.

14. Gribanov E.N., Oskotskaya E.R., Svensky S.I., Scientific notes of the Oryol State University. Series: Natural, technical and medical sciences, 2013, Vol. 53, No 3, pp. 121-123.

15. Rabo J., Chemistry of zeolites and catalysis on zeolites, Vol. 1, M., Mir, 1980. 506 p.

16. Lurie Yu.Yu. Analytical Chemistry Handbook, M., Chimiay, 1989, 448 p.

17. Mozgawa W, Handke M., Jastrzebski W., Journal of Molecular Structure, 2004, Vol. 704, No 1-3, pp. 247-257.

Gribanov Eugene N. - candidate of chemistry sciences, associate professor, Department of Chemistry, Turgrnev Orel State University, Orel, e-mail: gribanovEN@gmail.com 